\title{
Coulomb nuclear interference effect in dipion production in ultraperipheral heavy ion collisions
}

\author{
Yoshikazu Hagiwara, Cheng Zhang, Jian Zhou, and Ya-jin Zhou \\ Key Laboratory of Particle Physics and Particle Irradiation (MOE), \\ Institute of Frontier and Interdisciplinary Science, Shandong University, \\ (QingDao), Shandong 266237, China
}

(Received 22 December 2020; accepted 15 March 2021; published 19 April 2021)

\begin{abstract}
We study exclusive $\pi^{+} \pi^{-}$pair production near $\rho^{0}$ resonance peak in ultraperipheral heavy ion collisions. A pion pair can either be produced via two photon fusion process or from the decay of $\rho^{0}$ in photon-nuclear reaction. At very low pair transverse momentum, the electromagnetic and nuclear amplitudes become comparable. We show that the Coulomb nuclear interference amplitude gives rise to sizable $\cos \phi$ and $\cos 3 \phi$ azimuthal asymmetries, which can be used to constrain the phase of the dipolenucleus scattering amplitude.
\end{abstract}

DOI: 10.1103/PhysRevD.103.074013

\section{INTRODUCTION}

Strong electromagnetic fields induced by relativistic heavy ions can be effectively viewed as a flux of quasireal photons, which have been used to study a wide variety of physics in ultraperipheral heavy ion collisions (UPCs). Among many interesting UPC related topics, the diffractive vector mesons photoproduction on nuclei is one of the main focus of UPC physics as it offers access to the gluon tomography of nucleus as well as the tests of the CGC description of saturation physics [1-14]. There are also many active experimental programs devoted to the investigations of diffractive vector meson production [15-19] at both RHIC and LHC recently.

Experimentally, the vector meson $\rho^{0}$ events are reconstructed by measuring its decay product $\pi^{+} \pi^{-}$pair. The invariant mass spectrum of dipion can be well fitted with a relativistic Breit-Wigner resonance for $\rho^{0}$ plus a flat direct pion pair continuum. Such continuum background receives contributions from several different sources [20-23]: e.g., photon-Pomeron fusion process, and direct dipion production in photon-photon collisions. Since quasi-real photons are almost on shell, pion can be approximately treated as a pointlike scalar particle in the low invariant mass region of dipion system. As a result, the amplitude of $\gamma \gamma \rightarrow \pi^{+} \pi^{-}$ process can be straightforwardly computed using the scalar QED. The amplitude of the electromagnetic production is much smaller than that of the photonuclear interactions in

Published by the American Physical Society under the terms of the Creative Commons Attribution 4.0 International license. Further distribution of this work must maintain attribution to the author(s) and the published article's title, journal citation, and DOI. Funded by SCOAP ${ }^{3}$. most kinematic regions. However, this is not always the case, as the long range of the electromagnetic forces leads to rapidly growing amplitude with decreasing nuclear recoil momentum squared $t$, while the nuclear amplitude approaches a constant with vanishing $t$. At sufficiently low pair transverse momentum $\left(q_{\perp}\right)$, the pure electromagnetic and the nuclear amplitudes become comparable, and in particular, the Coulomb-nuclear interference (CNI) amplitude could be sizable.

Quite interestingly, we found that such CNI effect does not contribute to the azimuthal averaged cross section of the exclusive dipion production. Instead, it contributes to the azimuthal asymmetric cross section. To be more specific, the linear polarization of quasireal photons can induce distinctive $\cos \phi$ and $\cos 3 \phi$ modulations, where $\phi$ is defined as the azimuthal angle between $q_{\perp}$ and pion's transverse momentum $P_{\perp}$. The sign and the magnitude of these azimuthal asymmetries are sensitive to the relative phase between the EM amplitude and the QCD amplitude. These observables thus can be used to constrain the phase of photonuclear amplitude in future experiments. We noticed that the interference contribution between Odderon and Pomeron exchange leads to a different type asymmetry [24,25].

The key ingredient of our analysis presented in this paper is the linear polarization of quasi-real photons. This phenomenon was not recognized until very recently $[26,27]$. The linear polarization of coherent photons can give rise to a characteristic $\cos 4 \phi$ azimuthal asymmetry in pure EM dilepton production in UPCs [26-28], which later was clearly seen in the STAR measurement [29]. The computed impact parameter dependent asymmetry turns out to be in excellent agreement with the experimental data for the UPC case. With it being experimentally 
confirmed, the linearly polarized quasireal photon beam in heavy ion collisions can be used as a powerful tool to explore the novel QCD phenomenology. For example, the significant $\cos 2 \phi$ and $\cos 4 \phi$ asymmetries for $\rho^{0}$ meson production in UPCs have been reported by STAR collaboration previously [30]. The observed asymmetries result from incident photon's linear polarization. Its unique diffractive pattern crucially depends on transverse spacial distribution of the gluonic matter inside nucleus, and double slit like quantum interference effect [31].

The paper is organized as follows. In Sec. II, we derive all cross section formulas including pure EM dipion production contribution, those from the decay of $\rho^{0}$ meson and their interference terms. In Sec. III, we present the numerical estimations of $\cos \phi$ and $\cos 3 \phi$ azimuthal asymmetries for exclusive dipion production. The paper is summarized in Sec. IV.

\section{THE COULOMB NUCLEAR INTERFERENCE EFFECT}

The vector meson photoproduction is conventionally computed using the dipole model $[1,2]$, in which the whole process is divided into three steps: quasireal photon splitting into quark and antiquark pair, the color dipole scattering off nucleus, and subsequently recombining to form a vector meson after penetrating the nucleus target. Following this picture, one can directly write down the polarization averaged cross section of the vector meson production in ep or eA collisions. For the photoproduction of vector meson in UPCs, it is important to take into account double slit like quantum interference effect [32-34]. To this end, we developed a joint impact parameter dependent and $q_{\perp}$ dependent cross section formula in a previous work. The resulting unpolarized cross section gives excellent description to the STAR experimental data. We further computed the $\cos 2 \phi$ azimuthal asymmetry induced by the linear polarization of photons, and are able to describe its $q_{\perp}$ dependent behavior reasonably well [31].

One can easily derive the dipion production amplitude by multiplying the $\rho^{0}$ production amplitude with a simplified Breit-Wigner form which describes the transition from $\rho^{0}$ to $\pi^{+} \pi^{-}$,

$$
\begin{aligned}
\mathcal{M}_{\rho \rightarrow \pi^{+} \pi^{-}}= & i\left[\mathcal{A}_{c o}\left(x_{g}, \Delta_{\perp}\right)+\mathcal{A}_{\text {in }}\left(x_{g}, \Delta_{\perp}\right)\right] \\
& \times f_{\rho \pi \pi} \frac{P_{\perp} \cdot \epsilon_{\perp}^{\gamma}}{Q^{2}-M_{\rho}^{2}+i M_{\rho} \Gamma_{\rho}}
\end{aligned}
$$

where $\left[\mathcal{A}_{c o}\left(x_{g}, \Delta_{\perp}\right)+\mathcal{A}_{\text {in }}\left(x_{g}, \Delta_{\perp}\right)\right]$ represent $\rho^{0}$ production amplitude from coherent and incoherent scatterings. $\epsilon_{\perp}^{V}$ and $M_{\rho}$ are $\rho^{0}$ 's polarization vector and mass respectively. $Q$ is the invariant mass of dipion system and $P_{\perp}$ is defined as $P_{\perp}=\left(p_{1 \perp}-p_{2 \perp}\right) / 2$ with $p_{1 \perp}$ and $p_{2 \perp}$ being the produced pions' transverse momenta. $f_{\rho \pi \pi}$ is the effective coupling constant and fixed to be $f_{\rho \pi \pi}=12.24$ according to the optical theorem with the parameter $\Gamma_{\rho}=0.156 \mathrm{GeV}$. $\mathcal{A}_{c o}\left(\Delta_{\perp}\right)$ and $\mathcal{A}_{\text {in }}\left(\Delta_{\perp}\right)$ are given by,

$$
\begin{aligned}
& \mathcal{A}_{c o}\left(x_{g}, \Delta_{\perp}\right) \\
& =\int d^{2} b_{\perp} e^{-i \Delta_{\perp} \cdot b_{\perp}} \int \frac{d^{2} r_{\perp}}{4 \pi} N\left(r_{\perp}, b_{\perp}\right)\left[\Phi^{*} K\right]\left(r_{\perp}\right) \\
& \mathcal{A}_{\text {in }}\left(x_{g}, \Delta_{\perp}\right) \\
& =\sqrt{A} 2 \pi B_{p} e^{-B_{p} \Delta_{\perp}^{2} / 2} \\
& \quad \times\left[\int \frac{d^{2} r_{\perp}}{4 \pi} \mathcal{N}\left(r_{\perp}\right) e^{-2 \pi(A-1) B_{p} T_{A}\left(b_{\perp}\right) \mathcal{N}\left(r_{\perp}\right)}\left[\Phi^{*} K\right]\left(r_{\perp}\right)\right]
\end{aligned}
$$

which represent the coherent and incoherent vector meson production amplitudes respectively. $\mathcal{N}\left(r_{\perp}\right)$ is the dipolenucleon scattering amplitude. $N\left(r_{\perp}, b_{\perp}\right)$ is the elementary amplitude for the scattering of a dipole of size $r_{\perp}$ on a target nucleus at the impact parameter $b_{\perp}$ of the photon-nucleus collision. $T_{A}\left(b_{\perp}\right)$ is the nuclear thickness function. The longitudinal momentum fraction transferred to the vector meson via the dipole-nucleus interaction is given by $x_{g}=\sqrt{\frac{P_{\perp}^{2}+m_{\pi}^{2}}{S}}\left(e^{-y_{1}}+e^{-y_{2}}\right)$. And $\left[\Phi^{*} K\right]$ denotes the overlap of virtual photon wave function and the vector meson wave function,

$$
\begin{aligned}
{\left[\Phi^{*} K\right]\left(r_{\perp}\right)=} & \frac{N_{c} e e_{q}}{\pi} \int_{0}^{1} d z\left\{m_{q}^{2} \Phi^{*}\left(\left|r_{\perp}\right|, z\right) K_{0}\left(\left|r_{\perp}\right| e_{f}\right)\right. \\
& \left.+\left[z^{2}+(1-z)^{2}\right] \frac{\partial \Phi^{*}\left(\left|r_{\perp}\right|, z\right)}{\partial\left|r_{\perp}\right|} \frac{\partial K_{0}\left(\left|r_{\perp}\right| e_{f}\right)}{\partial\left|r_{\perp}\right|}\right\}
\end{aligned}
$$

where $z$ stands for the fraction of photon's light-cone momentum carried by quark. The physical meanings of all other parameters and shorthand notations appear in the above equations can be found in Ref. [31].

By recycling the result from our earlier work [31], it is straightforward to write down the differential cross section for dipion production from $\rho^{0}$ decay, 


$$
\begin{aligned}
\frac{d \sigma_{\rho \rightarrow \pi \pi}}{d^{2} p_{1 \perp} d^{2} p_{2 \perp} d y_{1} d y_{2} d^{2} \tilde{b}_{\perp}}= & \frac{1}{2(2 \pi)^{7}} \frac{P_{\perp}^{2}}{\left(Q^{2}-M_{\rho}^{2}\right)^{2}+M_{\rho}^{2} \Gamma_{\rho}^{2}} f_{\rho \pi \pi}^{2} \int d^{2} \Delta_{\perp} d^{2} k_{\perp} d^{2} k_{\perp}^{\prime} \delta^{2}\left(k_{\perp}+\Delta_{\perp}-q_{\perp}\right)\left(\hat{P}_{\perp} \cdot \hat{k}_{\perp}\right)\left(\hat{P}_{\perp} \cdot \hat{k}_{\perp}^{\prime}\right) \\
& \times\left\{\int d^{2} b_{\perp} e^{i \tilde{b}_{\perp} \cdot\left(k_{\perp}^{\prime}-k_{\perp}\right)}\left[T_{A}\left(b_{\perp}\right) \mathcal{A}_{\mathrm{in}}\left(x_{2}, \Delta_{\perp}\right) \mathcal{A}_{\mathrm{in}}^{*}\left(x_{2}, \Delta_{\perp}^{\prime}\right) \mathcal{F}\left(x_{1}, k_{\perp}\right) \mathcal{F}\left(x_{1}, k_{\perp}^{\prime}\right)+(A \leftrightarrow B)\right]\right. \\
& +\left[e^{i \tilde{b}_{\perp} \cdot\left(k_{\perp}^{\prime}-k_{\perp}\right)} \mathcal{A}_{c o}\left(x_{2}, \Delta_{\perp}\right) \mathcal{A}_{c o}^{*}\left(x_{2}, \Delta_{\perp}^{\prime}\right) \mathcal{F}\left(x_{1}, k_{\perp}\right) \mathcal{F}\left(x_{1}, k_{\perp}^{\prime}\right)\right] \\
& +\left[e^{i \tilde{b}_{\perp} \cdot\left(\Delta_{\perp}^{\prime}-\Delta_{\perp}\right)} \mathcal{A}_{c o}\left(x_{1}, \Delta_{\perp}\right) \mathcal{A}_{c o}^{*}\left(x_{1}, \Delta_{\perp}^{\prime}\right) \mathcal{F}\left(x_{2}, k_{\perp}\right) \mathcal{F}\left(x_{2}, k_{\perp}^{\prime}\right)\right] \\
& +\left[e^{i \tilde{b}_{\perp} \cdot\left(\Delta_{\perp}^{\prime}-k_{\perp}\right)} \mathcal{A}_{c o}\left(x_{2}, \Delta_{\perp}\right) \mathcal{A}_{c o}^{*}\left(x_{1}, \Delta_{\perp}^{\prime}\right) \mathcal{F}\left(x_{1}, k_{\perp}\right) \mathcal{F}\left(x_{2}, k_{\perp}^{\prime}\right)\right] \\
& \left.+\left[e^{i \tilde{b}_{\perp} \cdot\left(k_{\perp}^{\prime}-\Delta_{\perp}\right)} \mathcal{A}_{c o}\left(x_{1}, \Delta_{\perp}\right) \mathcal{A}_{c o}^{*}\left(x_{2}, \Delta_{\perp}^{\prime}\right) \mathcal{F}\left(x_{2}, k_{\perp}\right) \mathcal{F}\left(x_{1}, k_{\perp}^{\prime}\right)\right]\right\}
\end{aligned}
$$

where $y_{1}$ and $y_{2}$ are the final state pions' rapidities. $k_{\perp}, \Delta_{\perp}$, $k_{\perp}^{\prime}$, and $\Delta_{\perp}^{\prime}$ are incoming photon's transverse momenta and nucleus recoil transverse momenta in the amplitude and the conjugate amplitude respectively. $\tilde{b}_{\perp}$ is the transverse distance between the center of the two colliding nuclei. The unit transverse vectors are defined following the pattern as $\hat{k}_{\perp}=k_{\perp} /\left|k_{\perp}\right|$ and $\hat{P}_{\perp}=P_{\perp} /\left|P_{\perp}\right| . \mathcal{F}\left(x, k_{\perp}\right)$ describes the probability amplitude for finding a photon carries certain momentum. The squared $\mathcal{F}\left(x, k_{\perp}\right)$ is just the standard photon TMD distribution $f\left(x, k_{\perp}\right)$. At low transverse momentum it can be commonly computed with the equivalent photon approximation (also often referred to as the Weizsäcker-Williams method) which has been widely used to compute UPC observables (see for example [35-37]). In the equivalent photon approximation, $\mathcal{F}\left(x, k_{\perp}\right)$ reads,

$$
\mathcal{F}\left(x, k_{\perp}\right)=\frac{Z \sqrt{\alpha_{e}}}{\pi}\left|k_{\perp}\right| \frac{F\left(k_{\perp}^{2}+x^{2} M_{p}^{2}\right)}{\left(k_{\perp}^{2}+x^{2} M_{p}^{2}\right)}
$$

where $x$ is constrained according to $x=\sqrt{\frac{P_{\perp}^{2}+m_{\pi}^{2}}{S}}\left(e^{y_{1}}+e^{y_{2}}\right)$. $M_{p}$ is proton mass. $F$ is the nuclear charge form factor which will be specified later.

We now proceed to compute pion pair production amplitude in two photon collisions. The underlying production mechanism is quite complicated as discussed in Ref. [23]. However, since we focus on low invariant mass region, treating pion as a point like charged particle should be a good approximation. It is therefore justified to compute the production amplitude by using an effective lagrangian which is the simplest possible form satisfying the gauge invariance,

$$
\mathcal{L}_{I}=i e F_{\pi}\left(Q_{\gamma}^{2}\right) A^{\mu}\left[\left(\partial_{\mu} \pi^{\dagger}\right) \pi-\pi^{\dagger} \partial_{\mu} \pi\right]-e^{2} F_{\pi}\left(Q_{\gamma}^{2}\right) A_{\mu} A^{\mu} \pi^{\dagger} \pi
$$

where the EM form factor $F_{\pi}\left(Q_{\gamma}^{2}\right)$ can be simply approximated as 1 in the kinematic region under consideration.
With this effective coupling, one can readily derive the amplitude for the EM production of dipion,

$$
\mathcal{M}_{\gamma \gamma \rightarrow \pi \pi}=2 e^{2}\left[\epsilon_{\perp 1}^{\gamma} \cdot \epsilon_{\perp 2}^{\gamma}-\frac{2 P_{\perp}^{2}}{P_{\perp}^{2}+m_{\pi}^{2}}\left(\epsilon_{\perp 1}^{\gamma} \cdot \hat{P}_{\perp}\right)\left(\epsilon_{\perp 2}^{\gamma} \cdot \hat{P}_{\perp}\right)\right]
$$

Since the incident photons are linearly polarized, the polarization vectors $\epsilon_{\perp 2}$ and $\epsilon_{\perp 1}$ will be replaced with the corresponding photons transverse momenta respectively in the rest of calculations. Following the method outlined in Ref. [31], the impact parameter dependent cross section computed at the lowest order QED reads,

$$
\begin{aligned}
& \frac{d \sigma_{\gamma \gamma \rightarrow \pi \pi}}{d^{2} p_{1 \perp} d^{2} p_{2 \perp} d y_{1} d y_{2} d^{2} \tilde{b}_{\perp}} \\
& =\frac{\alpha_{e}^{2}}{Q^{4}} \frac{1}{(2 \pi)^{2}} \int d^{2} k_{\perp} d^{2} \Delta_{\perp} d^{2} k_{\perp}^{\prime} \delta^{2}\left(q_{\perp}-k_{\perp}-\Delta_{\perp}\right) e^{i\left(k_{\perp}-k_{\perp}^{\prime}\right) \cdot \tilde{b}_{\perp}} \\
& \quad \times 4\left[\hat{k}_{\perp} \cdot \hat{\Delta}_{\perp}-\frac{2 P_{\perp}^{2}}{P_{\perp}^{2}+m_{\pi}^{2}}\left(\hat{k}_{\perp} \cdot \hat{P}_{\perp}\right)\left(\hat{\Delta}_{\perp} \cdot \hat{P}_{\perp}\right)\right] \\
& \times\left[\hat{k}_{\perp}^{\prime} \cdot \hat{\Delta}_{\perp}^{\prime}-\frac{2 P_{\perp}^{2}}{P_{\perp}^{2}+m_{\pi}^{2}}\left(\hat{k}_{\perp}^{\prime} \cdot \hat{P}_{\perp}\right)\left(\hat{\Delta}_{\perp}^{\prime} \cdot \hat{P}_{\perp}\right)\right] \\
& \times \mathcal{F}\left(x_{1}, k_{\perp}^{2}\right) \mathcal{F}^{*}\left(x_{1}, k_{\perp}^{\prime}{ }^{2}\right) \mathcal{F}\left(x_{2}, \Delta_{\perp}^{2}\right) \mathcal{F}^{*}\left(x_{2}, \Delta_{\perp^{\prime}}^{2}\right)
\end{aligned}
$$

Here we only consider one photon exchange approximation. The multiple photon re-scattering effect is power suppressed in the invariant mass region near the $\rho^{0}$ resonance peak due to the involvement of the Weizsäcker-Williams type photon TMD in this process [37]. As a comparison, for the dipole type distribution, the photon TMD receives the significant Coulomb correction [38].

The interference term arises from the EM amplitude and the photon-nuclear scattering amplitude contributes to the cross section, 


$$
\begin{aligned}
& \frac{d \sigma_{I}}{d^{2} p_{1 \perp} d^{2} p_{2 \perp} d y_{1} d y_{2} d^{2} \tilde{b}_{\perp}} \\
& =\frac{\alpha_{e}}{Q^{2}} \frac{1}{(2 \pi)^{4}} \frac{1}{\sqrt{4 \pi}} \frac{2 M_{\rho} \Gamma_{\rho}\left|P_{\perp}\right| f_{\rho \pi \pi}}{\left(Q^{2}-M_{\rho}^{2}\right)^{2}+M_{\rho}^{2} \Gamma_{\rho}^{2}} \int d^{2} \Delta_{\perp} d^{2} k_{\perp} d^{2} k_{\perp}^{\prime} \delta^{2}\left(k_{\perp}+\Delta_{\perp}-q_{\perp}\right)\left[\hat{k}_{\perp} \cdot \hat{\Delta}_{\perp}-\frac{2 P_{\perp}^{2}}{P_{\perp}^{2}+m_{\pi}^{2}}\left(\hat{k}_{\perp} \cdot \hat{P}_{\perp}\right)\left(\hat{\Delta}_{\perp} \cdot \hat{P}_{\perp}\right)\right] \\
& \quad \times\left(\hat{P}_{\perp} \cdot \hat{k}_{\perp}^{\prime}\right) 2\left\{\left[e^{i \tilde{b}_{\perp} \cdot\left(k_{\perp}^{\prime}-k_{\perp}\right)} \mathcal{F}\left(x_{1}, k_{\perp}\right) \mathcal{F}\left(x_{2}, \Delta_{\perp}\right) \mathcal{F}\left(x_{1}, k_{\perp}^{\prime}\right) \mathcal{A}_{c o}^{*}\left(x_{2}, \Delta_{\perp}^{\prime}\right)\right]\right. \\
& \left.\quad+\left[e^{i \tilde{b}_{\perp} \cdot\left(\Delta_{\perp}^{\prime}-k_{\perp}\right)} \mathcal{F}\left(x_{2}, k_{\perp}\right) \mathcal{F}\left(x_{1}, \Delta_{\perp}\right) \mathcal{F}\left(x_{2}, k_{\perp}^{\prime}\right) \mathcal{A}_{c o}^{*}\left(x_{1}, \Delta_{\perp}^{\prime}\right)\right]\right\}
\end{aligned}
$$

In arriving at the above result, we have assumed that the dipole amplitude is purely imaginary. It is interesting to notice that due to the presence of angular structures $\left(\hat{k}_{\perp} \cdot \hat{\Delta}_{\perp}\right)\left(\hat{P}_{\perp} \cdot \hat{k}_{\perp}^{\prime}\right)$ and $\left(\hat{P}_{\perp} \cdot \hat{k}_{\perp}^{\prime}\right)\left(\hat{k}_{\perp} \cdot \hat{P}_{\perp}\right)\left(\hat{\Delta}_{\perp} \cdot \hat{P}_{\perp}\right)$, this interference term vanishes once carrying out the integration over the azimuthal angle of either $q_{\perp}$ or $P_{\perp}$. Therefore, it does not contribute to the azimuthal averaged cross section, but instead leads to $\cos \phi$ and $\cos 3 \phi$ azimuthal asymmetries. The emergencies of these nontrivial azimuthal correlations can be intuitively understood as follows: the linearly polarization state is the superposition of different helicity states. As illustrated in Fig. 1, supposing that two incoming photon both carry spin angular momentum 1 in the amplitude while the incident photon carries spin angular momentum -1 in the conjugate amplitude, the orbital angular momentum carried by the produced dipion system is 2 in the amplitude and -1 in the conjugate amplitude correspondingly. Such interference amplitude leads to a $\cos 3 \phi$ angular modulation. The similar argument applies to the $\cos \phi$ azimuthal asymmetry case.

\section{NUMERICAL ESTIMATIONS}

We now introduce all ingredients that are necessary for numerical calculations. Most of them follow our previous work [31]. We first introduce the parametrization for the $b_{\perp}$ dependent dipole-nucleus and dipole-nucleon scattering amplitudes. For the dipole-nucleus scattering amplitude, we adopt the parametrization $[5,6]$,

$$
N\left(b_{\perp}, r_{\perp}\right)=1-e^{-2 \pi B_{p} A T_{A}\left(b_{\perp}\right) \mathcal{N}\left(r_{\perp}\right)} .
$$

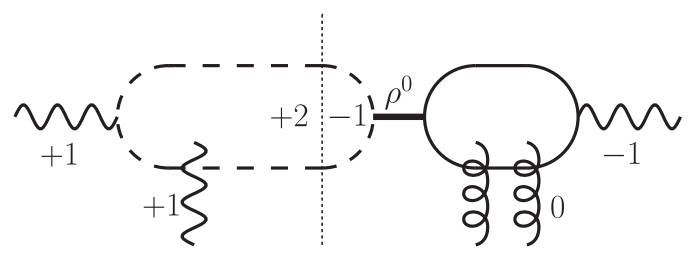

FIG. 1. An illustration of the mechanism giving rise to $\cos 3 \phi$ azimuthal asymmetry. The solid line represents the quark propagator, while the pion propagator is indicated by the dashed line.
Here the nuclear thickness function $T_{A}\left(b_{\perp}\right)$ is computed with the conventional Woods-Saxon distribution. The nuclear charge form factor is parametrized with the Woods-Saxon distribution as well,

$$
F\left(\vec{k}^{2}\right)=\int d^{3} r e^{i \vec{k} \cdot \vec{r}} \frac{C^{0}}{1+\exp \left[\left(r-R_{A}\right) / d\right]}
$$

where $R_{A}(\mathrm{Au}: 6.38 \mathrm{fm})$ is the radius and $d(\mathrm{Au}: 0.535 \mathrm{fm})$ is the skin depth. $C^{0}$ is a normalization factor. And $B_{p}=$ $4 \mathrm{GeV}^{2}$ in the IPsat model. For the dipole-nucleon scattering amplitude, we adopt a conventional parametrization, namely, the GBW model $[39,40]: \mathcal{N}\left(r_{\perp}\right)=1-e^{-\frac{Q_{s}^{2} r_{\perp}^{2}}{4}}$. For the scalar part of vector meson wave function, we use "Gaus-LC" wave function also taken from Refs. [5,6].

$$
\Phi^{*}\left(\left|r_{\perp}\right|, z\right)=\beta z(1-z) \exp \left[-\frac{r_{\perp}^{2}}{2 R_{\perp}^{2}}\right]
$$

with $\beta=4.47, R_{\perp}^{2}=21.9 \mathrm{GeV}^{-2}$ for $\rho$ meson.

UPC events are usually triggered at RHIC and LHC by detecting neutrons emitted at forward angles from a scattered nuclei. The probability for emitting a neutron is strongly dependent of the impact parameter. To account for this effect, one should integrate the differential cross section over impact parameter range from $2 R_{A}$ to $\infty$ with a weight function,

$$
2 \pi \int_{2 R_{A}}^{\infty} \tilde{b}_{\perp} d \tilde{b}_{\perp} P^{2}\left(\tilde{b}_{\perp}\right) d \sigma\left(\tilde{b}_{\perp}, \ldots\right)
$$

where the probability $P\left(\tilde{b}_{\perp}\right)$ of emitting a neutron from the scattered nucleus is conventionally parametrized as [41] $P\left(\tilde{b}_{\perp}\right)=P_{1 n}\left(\tilde{b}_{\perp}\right) \exp \left[-P_{1 n}\left(\tilde{b}_{\perp}\right)\right]$ which is denoted as the " 1 " event, while for emitting any number of neutrons (" $\mathrm{X}$ " event), the probability is given by $P\left(\tilde{b}_{\perp}\right)=$ $1-\exp \left[-P_{1 n}\left(\tilde{b}_{\perp}\right)\right]$ with $P_{1 n}\left(\tilde{b}_{\perp}\right)=5.45 * 10^{-5} \frac{Z^{3}(A-Z)}{A^{2 / 3} \tilde{b}_{\perp}^{2}} \mathrm{fm}^{2}$. All the subsequent numerical estimations are made for the "Xn" event. Having all these parameters specified, we are now ready to perform numerical calculation of the azimuthal asymmetries for dipion production in UPCs. 

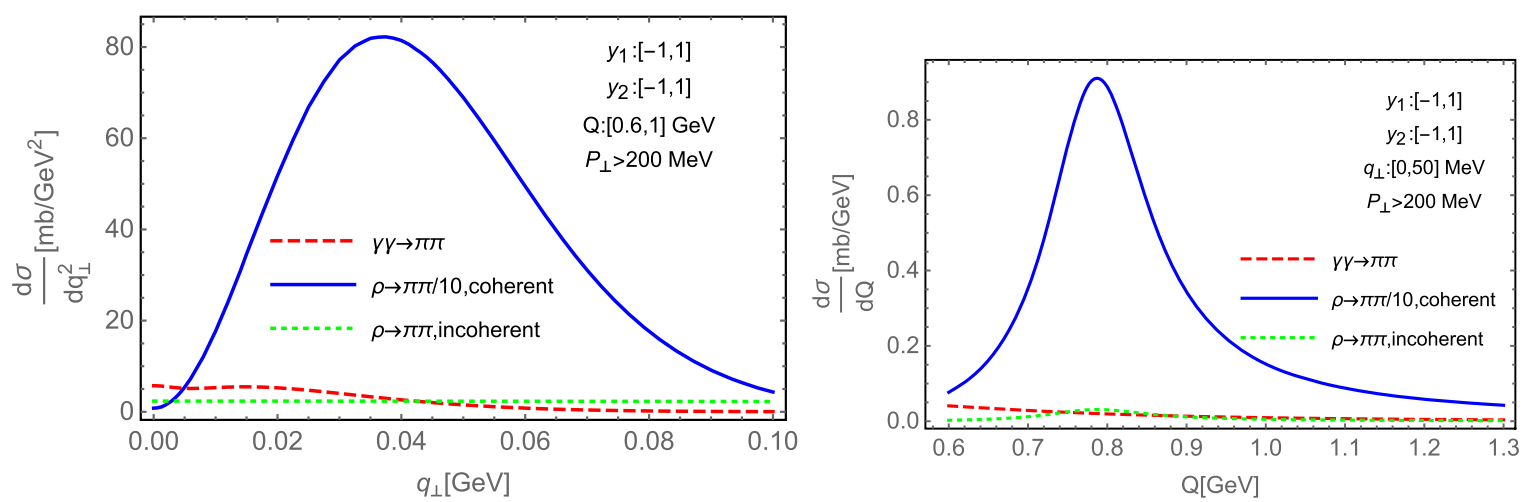

FIG. 2. The unpolarized differential cross section as the function of $q_{\perp}$ (left panel) and the invariant mass (right panel). The photonuclear cross section $d \sigma_{\rho \rightarrow \pi \pi}$ is rescaled by a factor $1 / 10$.
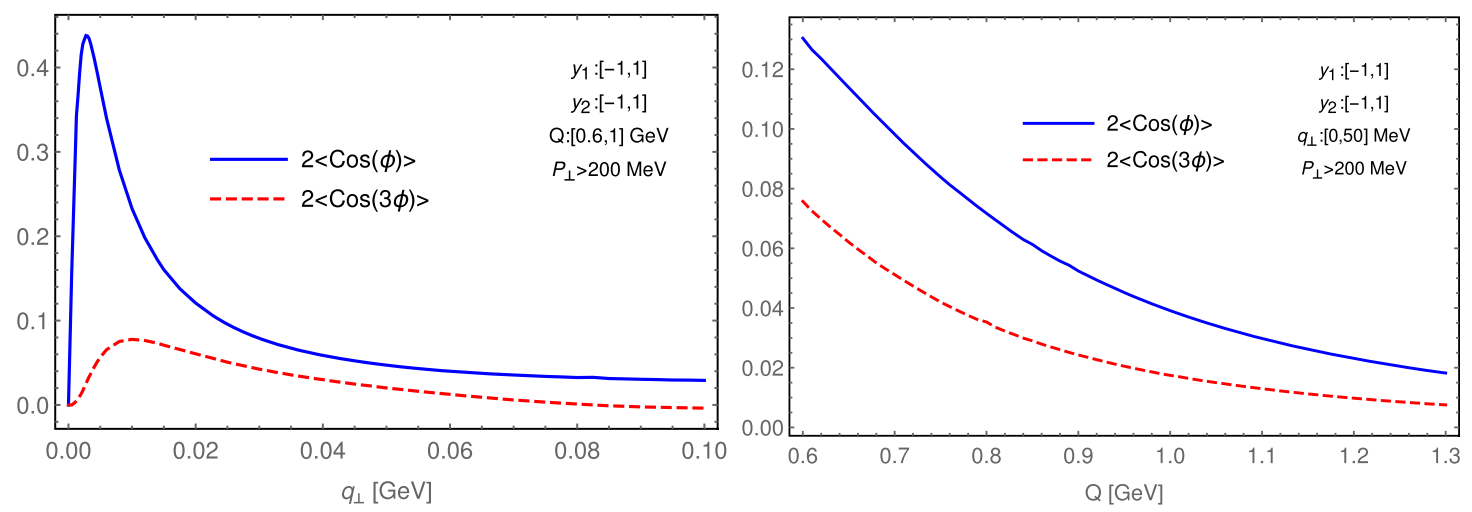

FIG. 3. $\cos \phi$ and $\cos 3 \phi$ azimuthal asymmetries as the function of $q_{\perp}$ (left panel) and the invariant mass (right panel).

We first present the contributions from the EM interaction and the photonuclear reaction to the azimuthal averaged cross section as the function of the invariant mass and pair transverse momentum in Fig. 2. One can see that the EM contribution is not negligible at low transverse momentum and in the low invariant mass region. It is worthy to emphasize again that the interference term drops out in the azimuthal averaged cross section. The suppression of the unpolarized cross section at low $q_{\perp}<40 \mathrm{MeV}$ is due to the destructive interference that was first discussed in Ref. [32].

The azimuthal asymmetries from the interference term are displayed in Fig. 3, where the asymmetries, i.e., the average value of $\cos 3 \phi$ and $\cos \phi$ are defined as,

$$
\langle\cos (n \phi)\rangle=\frac{\int \frac{d \sigma}{d \mathcal{P} . S .} \cos n \phi d \mathcal{P} . S .}{\int \frac{d \sigma}{d \mathcal{P} . S .} d \mathcal{P} . S .}
$$

with $n=1,3$. These asymmetries are calculated at the RHIC energy $\sqrt{S}=200 \mathrm{GeV}$. Both $\langle\cos \phi\rangle$ and $\langle\cos 3 \phi\rangle$ reach the maximal value at very low $q_{\perp}$ and then start to decrease with increasing $q_{\perp}$. They remain sizable and are roughly a few percentage levels in the transverse momentum region $20 \mathrm{MeV}<q_{\perp}<60 \mathrm{MeV}$. The analyzing power is basically of the same order of that from the helicity flip amplitude in elastic proton-proton scatterings, where the interference at low transverse momentum between the EM and hadronic contributions has long been used as a tool in the study of the phase of the hadronic amplitude [42,43] (see also a recent development [44]).

\section{CONCLUSION}

In summary, we have studied the $\cos \phi$ and $\cos 3 \phi$ azimuthal angular correlations in exclusive $\pi^{+} \pi^{-}$pair production near $\rho^{0}$ resonance peak in ultraperipheral heavy ion collisions, where $\phi$ is defined as the angle between pion's transverse momentum and pair's transverse momentum. The asymmetry essentially results from the linear polarization of incident coherent photons. We focus on low pair transverse momentum where the electromagnetic and nuclear amplitudes of dipion production are comparable. In this work, we demonstrate that $\cos \phi$ and $\cos 3 \phi$ azimuthal asymmetries are the characteristic signals of the CNI effect in exclusive pion pair production in UPCs. These azimuthal asymmetries are shown to be sizable in the kinematic region of interest, thus can serve as an alternative method to 
constrain the phase of the dipole amplitude. It should be feasible to measure the proposed observables at RHIC and LHC. We notice that the direct pion pair continuum receives contributions from several different channels [20-23]. Though they unlikely dominate low pair transverse momentum spectrum, it would be interesting to carry out a more comprehensive analysis in a future publication.

\section{ACKNOWLEDGMENTS}

J.Z. thanks Zhang-bu Xu, James Daniel Brandenburg and Chi Yang for helpful discussions. J.Z. has been supported by the National Natural Science Foundations of China under Grant No. 11675093. Y.Z. has been supported by the National Natural Science Foundations of China under Grant No. 11675092.
[1] M. Ryskin, Z. Phys. C 57, 89 (1993).

[2] S. J. Brodsky, L. Frankfurt, J. Gunion, A. H. Mueller, and M. Strikman, Phys. Rev. D 50, 3134 (1994).

[3] S. Klein and J. Nystrand, Phys. Rev. C 60, 014903 (1999).

[4] S. Munier, A. Stasto, and A. H. Mueller, Nucl. Phys. B603, 427 (2001).

[5] H. Kowalski and D. Teaney, Phys. Rev. D 68, 114005 (2003).

[6] H. Kowalski, L. Motyka, and G. Watt, Phys. Rev. D 74, 074016 (2006).

[7] M. Strikman, Nucl. Phys. B, Proc. Suppl. 179-180, 111 (2008).

[8] V. Rebyakova, M. Strikman, and M. Zhalov, Phys. Lett. B 710, 647 (2012).

[9] V. Guzey and M. Zhalov, J. High Energy Phys. 10 (2013) 207.

[10] T. Lappi and H. Mantysaari, Phys. Rev. C 83, 065202 (2011).

[11] Y.-P. Xie and X. Chen, Eur. Phys. J. C 76, 316 (2016).

[12] Y. Cai, W. Xiang, M. Wang, and D. Zhou, Chin. Phys. C 44, 074110 (2020).

[13] Y. Hagiwara, Y. Hatta, R. Pasechnik, M. Tasevsky, and O. Teryaev, Phys. Rev. D 96, 034009 (2017).

[14] Y. Hatta, M. Strikman, J. Xu, and F. Yuan, Phys. Lett. B 803, 135321 (2020).

[15] V. Khachatryan et al. (CMS Collaboration), Phys. Lett. B 772, 489 (2017).

[16] L. Adamczyk et al. (STAR collaboratio), Phys. Rev. C 96, 054904 (2017).

[17] J. Adam et al. (STAR Collaboration), Phys. Rev. Lett. 123, 132302 (2019).

[18] A. M. Sirunyan et al. (CMS Collaboration), Eur. Phys. J. C 79, 702 (2019).

[19] S. Acharya et al. (ALICE Collaboration), J. High Energy Phys. 06 (2020) 035.

[20] P. Soding, Phys. Lett. 19, 702 (1966).

[21] S. J. Brodsky, T. Kinoshita, and H. Terazawa, Phys. Rev. D 4, 1532 (1971).

[22] M. Klusek-Gawenda and A. Szczurek, Phys. Rev. C 87, 054908 (2013).

[23] A. Bolz, C. Ewerz, M. Maniatis, O. Nachtmann, M. Sauter, and A. Schöning, J. High Energy Phys. 01 (2015) 151.
[24] P. Hägler, B. Pire, L. Szymanowski, and O. V. Teryaev, Phys. Lett. B 535, 117 (2002); 540, 324(E) (2002).

[25] P. Hagler, B. Pire, L. Szymanowski, and O. V. Teryaev, Eur. Phys. J. C 26, 261 (2002).

[26] C. Li, J. Zhou, and Y.-J. Zhou, Phys. Lett. B 795, 576 (2019).

[27] C. Li, J. Zhou, and Y.-J. Zhou, Phys. Rev. D 101, 034015 (2020).

[28] B.-W. Xiao, F. Yuan, and J. Zhou, Phys. Rev. Lett. 125, 232301 (2020).

[29] J. Adam et al. (STAR Collaboration), arXiv:1910.12400.

[30] J. D. Brandenburg et al. (STAR Collaboration), Proceedings of the Quark Matter 2019, Wuhan, China (2019), https:// indico.cern.ch/event/792436/contributions/3535864/.

[31] H. Xing, C. Zhang, J. Zhou, and Y.-J. Zhou, J. High Energy Phys. 10 (2020) 064.

[32] S. R. Klein and J. Nystrand, Phys. Rev. Lett. 84, 2330 (2000).

[33] B. Abelev et al. (STAR Collaboration), Phys. Rev. Lett. 102, 112301 (2009).

[34] W. Zha, L. Ruan, Z. Tang, Z. Xu, and S. Yang, Phys. Rev. C 99, 061901 (2019).

[35] S. Klein, A. Mueller, B.-W. Xiao, and F. Yuan, Phys. Rev. Lett. 122, 132301 (2019).

[36] W. Zha, J. D. Brandenburg, Z. Tang, and Z. Xu, Phys. Lett. B 800, 135089 (2020).

[37] S. Klein, A. Mueller, B.-W. Xiao, and F. Yuan, Phys. Rev. D 102, 094013 (2020).

[38] Z.-H. Sun, D.-X. Zheng, J. Zhou, and Y.-J. Zhou, Phys. Lett. B 808, 135679 (2020).

[39] K. J. Golec-Biernat and M. Wusthoff, Phys. Rev. D 59, 014017 (1998).

[40] K. J. Golec-Biernat and M. Wusthoff, Phys. Rev. D 60, 114023 (1999).

[41] G. Baur, K. Hencken, and D. Trautmann, J. Phys. G 24, 1657 (1998).

[42] N. H. Buttimore, E. Gotsman, and E. Leader, Phys. Rev. D 18, 694 (1978); 35, 407(E) (1987).

[43] N. H. Buttimore, B. Kopeliovich, E. Leader, J. Soffer, and T. Trueman, Phys. Rev. D 59, 114010 (1999),

[44] Y. Hagiwara, Y. Hatta, R. Pasechnik, and J. Zhou, Eur. Phys. J. C 80, 427 (2020). 